\title{
Significado y sentido en el aprendizaje escolar. Reflexiones en torno al concepto de aprendizaje significativo
}

\author{
CÉSAR COLL \\ Universidad de Barcelone

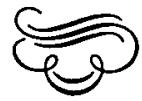 \\ Resumen
}

El concepto de aprendizaje significativo es un instrumento potencialmente ütil y valioso para el análisis y la reflexión psicopedagógica. Sin embargo, para que pueda desempeñar adecuadamen. te este papel, es necesario despojarlo de muchas de las connotaciones que ba ido acumulando de forma subrepticia y, al mismo tiempo, desarrollar otras que hasta el momento han sido escasamente tomadas en consideración. En concreto, se sugiere atender tanto al sentido como al significado del aprendizaje escolar; renunciar a las connotaciones mäs individualistas del proceso de construc. ción de significados y sentidos; $y$, por último, resituar este proceso de construcción en el contexto de relación y comunicación interpersonal que es intrínseco al acto de enseñanza.

Palabras clave: Significado, Sentido, Aprendizaje escolar, Aprendizaje significativo.

\section{Meaning and sense in school learning. Thoughts about meaningful learning}

\begin{abstract}
The concept of meaningful learning is a potentially useful and valuable instrument for the psychopedagogical reflection and analysis. If we want it to play that nole we need to free it from all those connotations it has surreptitiously accumulated and, at the same time, develop others which for the moment have been very little taken into consideration. Specifically, it is suggested to pay attention to the sense as well as to the meaning of school learning; to renounce to the more individual connotations of the process of meaning and sense construction and, finally, to replace this process of construction into the context of interpersonal communication and relationship inherent to the act of teaching.
\end{abstract}

Key words: Meaning, Sense, School learning, Meaningful learning.

Dirección del autor: Universidad de Barcelona. Departamento de Psicología Evolutiva y de la Educación. Adolf Florensa, s/n. 08028 Barcelona. 
Existe actualmente una coincidencia en subrayar, desde concepciones y enfoques psicopedagógicos relativamente dispares, la importancia del aprendizaje significativo como elemento clave de la educación escolar. Se insiste en que únicamente los aprendizajes significativos consiguen promover el desarrollo personal de los alumnos; se valoran las propuestas didácticas y las actividades de aprendizaje en función de su mayor o menor potencialidad para promover aprendizajes significativos; se proponen procedimientos y técnicas de evaluación susceptibles de detectar el grado de significatividad de los aprendizajes realizados, etcétera. Esta coincidencia es sin duda sorprendente en la medida en que la prácticas educativas, tanto las escolares como las extraescolares, constituyen un ámbito de conocimiento y de actividad profesional más bien propenso a polé- micas y a puntos de vista encontrados.

En realidad la idea, o mejor dicho, algunas de las ideas que subyacen al uso actual del concepto de aprendizaje significativo cuentan con numerosos antecedentes en la historia del pensamiento educativo. Podemos remontarnos, en primer lugar, a la tradición puerocentrista de los movimientos pedagógicos renovadores de principios de siglo, que hunde sus raíces en el pensamiento de Rousseau y a la que pertenecen autores tan destacados como Claparède, Dewey, Ferrière, Montessori, Decroly, Cousinet, Freinet y otros muchos que, más allá de las diferencias entre sus respectivos planteamientos, comparten el principio de autoestructuración del conocimiento, es decir, ven al alumno como el verdadero agente y el responsable último de su propio proceso de aprendizaje, como «el artesano de su propia construcción» (Not, 1979). En segundo lugar, cabe mencionar la tradición, más reciente, de la hipótesis del aprendizaje por descubrimiento desarrollada en los años sesenta y de las propuestas pedagógicas que defienden el principio de que el alumno adquiera el conocimiento con sus propios medios, o como afirma Bruner en su conocido trabajo sobre el acto de descubrimiento, «mediante el uso de su propia mente» (Bruner, 1961). En tercer lugar podemos citar las propuestas pedagógicas inspiradas en las tesis constructivistas de Piaget sobre la naturaleza y el desarrollo de la inteligencia, tesis que él mismo proponía sintetizar en la siguiente afirmación elevada a «principio fundamental de los métodos activos: comprender es inventar o reconstruir por reinvención» (Piaget, 1974).

No se agotan, sin embargo, con estas referencias, ciertamente ricas y variadas, los antecedentes del concepto de aprendizaje significativo tal como es utilizado actualmente en el discurso y en la práctica pedagógica. En una tradición de pensamiento distinta de las anteriores encontramos, por ejemplo, los estudios e investigaciones sobre la curiosidad epistémica y la actividad exploratoria en el marco de las teorías de la motivación. Dado que, según los postulados de la teoría de la activación (arousal) formulada por Hebb y Berlyne en los años sesenta, la motivación por explorar, descubrir, aprender y comprender está presente en mayor o menor grado en todas las personas, la actividad exploratoria se convierte en un poderoso instrumento para la adquisición de nuevos conocimientos. Desde un punto de vista pedagógico, esto conduce a la propuesta de confrontar al alumno con situaciones que poseen una serie de características (novedad, complejidad, ambigüedad, incongruencia, etc.) susceptibles de activar la motivación intrínseca y, de este modo, provocar una curiosidad epistémica y una actividad exploratoria dirigida a reducir el conflicto conceptual, la incertidumbre y la tensión generada por las características de la situación (FarnhamDiggory, 1972). 
Permítasenos citar todavía otro antecedente que muestra hasta qué punto el concepto de aprendizaje significativo es depositario de ideas y connotaciones que tienen su origen en enfoques distintos, no siempre totalmente compatibles, del psiquismo humano. Nos referimos a la concepción humanística del aprendizaje que está en la base de propuesta formulada por Rogers (1969), de enseñanza no directiva o de enseñanza centrada en el alumno. Esta propuesta, que se caracteriza, entre otras cosas, por recoger la aspiración ancestral de una educación adaptada a las necesidades de cada individuo, sitúa el desarrollo personal del alumno en el centro del proceso educativo y señala como fin prioritario de la educación que la persona funcione de manera integrada y efectiva, que construya su propia realidad, que encuentre su identidad particular. Recuérdese, por ejemplo, la insistencia de los autores humanistas en aprender a percibir, a conocer, a sentir la vida (feeling life) y la propia identidad como objetivos fundamentales de la educación.

Pero es quizás en la crítica que los autores humanistas dirigen al «aprendizaje extrínsecos y en las alternativas que proponen al mismo donde se encuentra un mayor paralelismo con el concepto de aprendizaje significativo. Maslow (1968) denomina aprendizaje extrínseco a la adquisición de contenidos externos a la persona, impuestos culturalmente, ajenos a su identidad y que poco o nada tienen que ver con lo que hay de peculiar, de idiosincrásico, de definitorio, en cada ser humano. La mayor parte de las teorías del aprendizaje y de los modelos educativos reposan, según este autor, sobre una concepción extrínseca del aprendizaje, ignorando sistemáticamente los valores, fines, sentimientos y actitudes del alumno. De aquí que la educación que se imparte habitualmente en los centros escolares sea impersonal, centrada en el enseñante, extrínseca, utilitaria, directiva y, en último término, irrelevante para las necesidades individuales del alumno. Frente al aprendizaje extrínseco, hay otro, que ocurre en gran medida al margen del sistema escolar, y que surge directamente de las experiencias personales. Es a través de estas experiencias personales, de una serie de aprendizajes fundamentalmente intrínsecos, como aprendemos más sobre nosotros mismos y llegamos a descubrir y reconstruir nuestra propia identidad. A partir de aquí, las orientaciones para erradicar el aprendizaje extrínseco de la educación formal son bien conocidas: que los alumnos decidan por sí mismos lo que quieren aprender, pues sólo ellos pueden saber lo que se adapta mejor a su individualidad, a sus necesidades básicas; dar prioridad al objetivo de aprender a aprender frente al objetivo de aprender destrezas o contenidos; practicar la autoevaluación como la única forma de evaluación relevante; prestar una aten. ción especial a la educación de la sensibilidad y de los sentimientos; eliminar cualquier componente amenazador de las situaciones de aprendizaje, etcétera.

Este breve rastreo de antecedentes, que de ningún modo puede considerarse exhaustivo, muestra con claridad que la coincidencia en subrayar la importancia del aprendizaje significativo como elemento clave de la educación escolar puede ser un tanto engañosa. En efecto, su utilización desde enfoques y planteamientos psicopedagógicos relativamente dispares, lejos de representar una unanimidad conceptual, recubre más bien concepciones distintas no siempre compatibles del aprendizaje escolar y de la manera de ejercer la influencia educativa. La polisemia del concepto, la diversidad de significaciones que ha ido acumulando, explica en gran parte su atractivo y su utilización generalizada y obliga, al mismo tiempo, a mantener una prudente reserva.

Conviene subrayar que, hoy por hoy, es un concepro que no posee una signi- 
ficación unívoca, como puede comprobarse en el uso indiscriminado y acrítico del mismo en lo que va camino de convertirse en una moda más de las que periódicamente y de forma recurrente invaden el campo de la educación. Frente a su utilización, a todas luces incorrecta, como fórmula mágica susceptible de mediar en la resolución de los problemas educativos de naturaleza más variada - la elaboración de materiales didácticos, el diseño de actividades de aprendizaje y la evaluación de resultados, la transferencia de los aprendizajes escolares, etc.-, el concepto de aprendizaje significativo posee, a nuestro juicio, un gran valor heurístico y encierra una enorme potencialidad como instrumento de análisis, de reflexión y de intervención psicopedagógica. Su interés no reside, pues, tanto en las soluciones que aporta como en la nueva perspectiva que conduce a adoptar en el estudio de los procesos de enseñanza/aprendizaje. Es precisamente este valor heurístico, esta potencialidad como instrumento de análisis y de reflexión, la que intentaremos ilustrar seguidamente indagando tres aspectos o componentes del concepto de aprendizaje significativo que nos pueden permitir avanzar hacia una delimitación más precisa del mismo.

\section{APRENDIZAJE ESCOLAR Y CONSTRUCCION DE SIGNIFICADOS}

Hablar de aprendizaje significativo equivale, ante todo, a poner de relieve el proceso de construcción de significados como elemento central del proceso de enseñanza/aprendizaje. El alumno aprende un contenido cualquiera - un concepto, una explicación de un fenómeno físico o social, un procedimiento para resolver determinado tipo de problemas, una norma de comportamiento, un valor a respetar, etc. - cuando es capaz de atribuirle un significado. De hecho, en sentido estricto, el alumno puede aprender también estos contenidos sin atribuirles significado alguno; es lo que sucede cuando los aprende de una forma puramente memorística y es capaz de repetirlos o de utilizarlos mecánicamente sin entender en absoluto lo que está diciendo o lo que está haciendo.

La mayoría de las veces, sin embargo, lo que sucede es que el alumno es capaz de atribuir únicamente significados parciales a lo que aprende: el concepto aprendido - o la explicación, o el valor, o la norma de conducta, o el procedimiento de resolución de problemas - no significa exactamente lo mismo para el profesor que lo ha enseñado que para el alumno que lo ha aprendido, no tiene las mismas implicaciones ni el mismo poder explicativo para ambos, que no pueden utilizarlo o aplicarlo en igual extensión y profundidad; en suma, no posee para ellos la misma fuerza como instrumento de comprensión y de acción sobre la parcela de la realidad a la que se refiere. Quiere decir esto que la significatividad del aprendizaje no es una cuestión de todo o nada, sino más bien de grado; en consecuencia, en vez de proponernos que los alumnos realicen aprendizajes significativos, quizás sería más adecuado intentar que los aprendizajes que llevan a cabo sean, en cada momento de la escolaridad, lo más significativos posible. Lejos de ser un juego de palabras, este cambio de perspectivas es importante porque subraya el carácter abierto y dinámico del aprendizaje escolar y plantea el problema de la dirección o direcciones en las que debe actuar la enseñanza para que los alumnos profundicen y amplíen los significados que construyen mediante su participación en las actividades de aprendizaje.

Pero, ¿qué quiere decir exactamente que los alumnos construyen significados? Una primera aproximación, sin lugar a duda la más conocida, es la que 
proporcionan Ausubel y sus colaboradores (ver, por ejemplo, Ausubel, Novak y Hanesian, 1983; Novak, 1982). Siguiendo a estos autores, construimos significados cada vez que somos capaces de establecer relaciones «sustantivas y no arbitrarias entre lo que aprendemos y lo que ya conocemos. Así, la mayor o menor riqueza de significados que atribuiremos al material de aprendizaje dependerá de la mayor o menor riqueza y complejidad de las relaciones que seamos capaces de establecer. Por ejemplo, la observación de la fauna y la flora de una región cualquiera dará lugar a la construcción de significados distintos en el caso de un alumno que no posee conocimientos previos de biología, en el caso de un alumno que sí posee algún tipo de conocimientos de este tipo y que, por lo tanto, puede establecer múltiples relaciones de similitud y de contraste, o en el caso de un alumno que además puede relacionar lo observado con las actividades económicas, las formas de habitat y las costumbres de los habitantes de la región. En los tres casos, el alumno en cuestión atribuye significados a lo que observa, pero estos significados tienen una amplitud y una riqueza netamente distinta.

En términos piagetianos, podríamos decir que construimos significados integrando o asimilando el nuevo material de aprendizaje a los esquemas que ya poseemos de comprensión de la realidad. Lo que presta un significado al material de aprendizaje es precisamente su asimilación, su inserción, en estos esquemas previos. En un caso límite, lo que no podemos asimilar a ningún esquema previo carece totalmente de significado para nosotros. La experiencia cotidiana nos informa que podemos estar en contacto con multitud de hechos, de fenómenos y de situaciones que no existen prácticamente para nosotros, que no significan nada, hasta que, por la razón que sea, se insertan en nuestros esquemas de actuación o de conocimiento adquiriendo de golpe un significado hasta ese momento desconocido. Pero siguiendo con la terminología piagetiana, la construcción de significados implica igualmente una acomodación, una diversificación, un enriquecimiento, una mayor interconexión de los esquemas previos. Al relacionar lo que ya sabemos con lo que estamos aprendiendo, los esquemas de acción y de conocimiento - lo que ya sabemos - se modifican y, al modificarse, adquieren nuevas potencialidades como fuente futura de atribución de significados.

Hemos mencionado ya que no siempre el aprendizaje es significativo, es decir, que no siempre da lugar a la construcción de significados. En muchas ocasiones, el aprendizaje se limita a la mera repetición memorística. De hecho, es más bien difícil alcanzar un nivel elevado de significatividad en el aprendizaje escolar. Las condiciones que exige su realización no son siempre fáciles de cumplir. Ausubel y sus colaboradores han insistido en numerosas ocasiones sobre las exigencias que plantea el aprendizaje significativo. Ante todo, es necesario que el nuevo material de aprendizaje, el contenido que el alumno va a aprender, sea potencialmente significativo, es decir, sea susceptible de dar lugar a la construcciọn de significados. Para ello, debe cumplir dos condiciones, una intrínseca al propio contenido de aprendizaje y la otra relativa al alumno particular que va a aprenderlo.

La primera condición es que el contenido posea una cierta estructura interna, una cierta lógica intrínseca, un significado en sí mișmo. Difícilmente el alumno podrá construir significados si el contenido de aprendizaje es vago, está poco estructurado o es arbitrario; es decir, si no es potencialmente significativo desde el punto de vista lógico. Obviamente, esta potencial significatividad lógica, co- 


\section{6}

mo la denomina Ausubel, no depende sólo de la estructura interna del contenido, sino también de la manera como éste se le presenta al alumno. Así, por ejemplo, un contenido como el uso de las preposiciones en inglés presenta en principio una escasa significatividad lógica, al menos para un castellanoparlante; sin embargo, puede ser presentado a los alumnos de tal manera que su significarividad lógica quede ampliamente realzada ( $\mathrm{Pla}, 1987)$. Pero no basta con que el contenido posea significatividad lógica. Se requiere todavía una segunda condición: para que un alumno determinado construya significados a propósito de este contenido es necesario, además, que pueda ponetlo en relación de forma no arbitraria con la que ya conoce, que pueda asimilarlo, que pueda insertarlo en las redes de significados ya construidas en el transcurso de sus experiencias previas de aprendizaje; en otros términos, es necesario que el contenido sea potencialmente significativo desde el punto de vista psicológico. Esta potencial significatividad psicológica del material de aprendizaje explica, por otra parte, la importancia acordada por Ausubel y sus colaboradores al conocimiento previo del alumno como el factor decisivo en el momento de afrontar la adquisición de nuevos conocimientos.

La potencial significatividad lógica y psicológica del contenido de aprendizaje, con ser dos condiciones necesarias no son, sin embargo, todavía suficientes para que el alumno construya significados. Es necesario, además, que éste, el alumno, tenga una actitud favorable para aprender significativamente. Este requisito, a menudo olvidado en las discusiones sobre el tema que nos ocupa, es una consecuencia lógica del protagonismo del alumno y de su responsabilidad en el aprendizaje. La actitud favorable hacia el aprendizaje significativo hace referencia a una intencionalidad del alumno para. relacionar el nuevo material de aprendizaje con lo que ya conoce, con los conocimientos adquiridos previamente, con los significados ya construidos. Cuando la intencionalidad es escasa, el alumno se limitará probablemente a memorizar lo aprendido de una forma un tanto mecánica y repetitiva; por el contrario, cuando la intencionalidad es elevada, el alumno establecerá múltiples y variadas relaciones entre lo nuevo y lo que ya conoce. El que un alumno se sitúe en uno u otro lugar del continuo que delimitan estos dos extremos va a depender, en definitiva, de su motivación para aprender significativamente y de la habilidad del profesor para despertar e incrementar esta motivación. La intervención del profesor en este sentido es un factor determinante, pues la memorización mecánica y repetitiva de lo aprendido suele aparecer en principio como un procedimiento mucho más cómodo y económico en tiempo y energía para el alumno que la construcción de significados mediante la búsqueda y el establecimiento de relaciones sustantivas entre lo nuevo y lo que ya conoce.

Lo dicho hasta aquí basta para darse cuenta de que el aprendizaje significativo de un contenido cualquiera implica inevitablemente su memorización comprensiva, su ubicación o almacenamiento en una red más o menos amplia de significados. Asimismo, en la medida en que contribuye a ampliar y extender dicha red de significados, se incrementa la capacidad del alumno para establecer nuevas relaciones cuando se enfrente a posteriores tareas o situaciones, por lo que un aprendizaje realizado de forma significativa es, al mismo tiempo, un aprendizaje que tiene un elevado valor funcional, es decir, un aprendizaje útil, un aprendizaje que puede ser utilizado con relativa facilidad para generar nue: vos significados.

Dos últimos comentarios para terminar con este apartado. En primer lugar, 
el concepto de aprendizaje significativo, tal como aparece en las formulaciones de Ausubel y de sus colaboradores, implica un cambio de perspectiva en la solución dada al clásico problema pedagógico de la preparación o disponibilidad (readiness) para el aprendizaje escolar. El énfasis ya no reside en la competencia intelectual del alumno, directa o indirectamente relacionada con su nivel de desarrollo evolutivo, sino más bien en la existencia de conocimientos previos pertinentes para el contenido a aprender, que dependen, por supuesto, en parte de dicha competencia intelectual, pero también, y sobre todo, de las experiencias previas de aprendizaje, tanto escolares como extraescolares.

Pero el concepto de aprendizaje significativo supone, ante todo, un cambio de perspectiva radical en la manera de entender el proceso de enseñanza/aprendizaje. Frente a la concepción tradicional y habitual de que el aprendizaje del alumno depende directamente de la influencia del profesor y de la metodología de enseñanza utilizada, se pone de reliève la importancia del conocimiento previo del alumno y, en general, de sưs procesos de pensamiento. Estos procesos devienen así el elemento mediador entre, por una parte, los procedimientos instruccionales o didácticos y, por otra, los resultados del aprendizaje. La construcción de significados que lleva a cabo el alumno a partir de la enseñanza es el elemento mediador susceptible de explicar los resultados de aprendizaje finalmente obtenidos.

Ahora bien, como ha puesto de relieve Wittrock (1986), en una revisión reciente sobre los procesos de pensamiento del alumno, existe evidencia empírica para afirmar que, junto al conocimiento previo, existen otros aspectos o procesos psicológicos que actúan como mediadores entre la enseñanza y los resultados del aprendizaje: la percepción que tiene el alumno de la escuela, del profesor y de sus actuaciones; sus expectativas ante la enseñanza; sus motivaciones, creencias, actitudes y atribuciones; las estrategias de aprendizaje que es capaz de utilizar, etc. En definitiva, todo parece indicar que el alumno construye significaciones al mismo tiempo que atribuye un sentido a lo que aprende, de tal manera que las significaciones que finalmente construye a partir de lo que se le enseña no depende sólo de los conocimientos previos que posea y de su puesta en relación con el nuevo material de aprendizaje, sino que también del sentido que atribuye a éste y a la propia actividad de aprendizaje.

\section{SIGNIFICADO Y SENTTDO EN EL APRENDIZAJE ESCOLAR}

Utilizamos el término asentido» con el fin de subrayắr el carácter experiencial que, en buena lógica constructivista, impregna el apréndizaje escolar. La percepción que tiene el alumno de una actividad concreta y particular de aprendizaje no coincide necesariamente con la que tiene el profesor; los objetivos del profesor y el alumno, sus intenciones y sus motivaciones al proponerla y participar en ella, son a menudo diferentes. Hay, pues, todo un conjunto de factores, que podríamos calificar como motivaciones, relacionales o incluso afectivos, que desempeñan un papel de primer orden en la movilización de los conocimientos previos del alumno y sin cuya consideración es imposible entender los significados que el alumno construye a propósito de los contenidos que se le enseñan en la escuela. Una interpretación radicalmente constructivista del concepto de aprendizaje significativo obliga a ir más allá de la simple consideración de los procesos cognoscitivos del alumno como elemento mediador de la enseñanza. 


\section{8}

La construcción de significados implica al alumno en su totalidad y no sólo sus conocimientos previos y su capacidad para establecer relaciones sustantivas entre éstos y el nuevo material de aprendizaje, o entre las diferentes partes del material de aprendizaje como ha señalado el propio Wittrok (1974), en su modelo de «aprendizaje generativo».

Aunque por el momento poseemos una comprensión muy limitada de los procesos psicológicos mediante los cuales los alumnos atribuyen un sentido a las actividades de aprendizaje, no cabe ninguna duda acerca de su existencia y de su importancia para la realización de aprendizajes significativos. Son enormemente ilustrativos a este respecto los trabajos de Marton y sus colaboradores, de la Universidad de Gothenburg (Marton, 1981, 1983) y los de Entwistle y sus colaboradores, de la Universidad de Edimburgo (Entwistle y Ramsden, 1983; Entwistle, 1987). En una serie de investigaciones dirigidas a estudiar el aprendizaje desde la perspectiva de los propios alumnos - concretamente de alumnos de enseñanza superior-, estos autores han identificado hasta tres maneras típicas de abordar o enfocar las tareas de aprendizaje que denominan respectivamente enfoque en profundidad (deep approach), enfoque superficial (surface approach) y enfoque estratégico (strategic approach).

El primero presenta fuertes similitudes con la disposición a realizar aprendizajes altamente significativos, puesto que se caracteriza, entre otros extremos, porque los alumnos muestran un elevado grado de implicación en el contenido, intentan profundizar al máximo en su comprensión y exploran sus posibles relaciones e interconexiones con conocimientos previos y experiencias personales. El segundo, por el contrario, presenta una cierta similitud con la tendencia descrita por Ausubel a realizar aprendizajes poco significativos y un tanto repetitivos o mecánicos; los alumnos que adoptan este enfoque ante una tarea determinada se preocupan ante todo por memorizar la información cuyo recuerdo, suponen, será evaluado posteriormente, por atenerse de forma estricta a las exigencias o instrucciones proporcionadas para su realización, por no interrogarse acerca de los objetivos o la finalidad de la tarea, por centrarse en aspectos parciales de la misma, y por una cierta incapacidad para distinguir los aspectos esenciales de los accesorios o circunstanciales. El enfoque estratégico, por último, se caracteriza por el intento de alcanzar el máximo rendimiento posible en la realización de la tarea mediante la planificación cuidadosa de las actividades, del material necesario, de los esfuerzos y del tiempo disponible.

Al margen del interés que supone el paralelismo existente entre, por una parte, las descripciones que proporcionan los alumnos de sus propios procesos de aprendizaje y, por otra, las descripciones que proporcionan los psicólogos, el hecho más importante que han puesto de manifiesto estos trabajos es, como señala acertadamente Entwistle, que la adopción de uno u otro enfoque depende, en último término, de la intención con la que el alumno se enfrenta a la tarea concreta de aprendizaje. Una misma tarea, presentada de forma idéntica a un grupo de alumnos, dará lugar a la adopción de enfoques de aprendizaje distintos, según que la intención de éstos se dirija preferentemente a buscar y establecer conexiones con sus conocimientos previos y sus experiencias personales (enfoque en profundidad), a memorizar elementos discretos de información (enfoque superficial), o a rentabilizar al máximo el esfuerzo y el tiempo disponible (enfoque estratégico). Un mismo alumno, por otra parte, puede adoptar sucesivamente uno u otro enfoque de aprendizaje según con la intención que lleve a cabo las respectivas tareas.: En definitiva, la misma enseñanza dirigida 
a un grupo de alumnos puede dar lugar a interpretaciones muy diferentes y, consecuentemente, a la construcción de significados igualmente muy distintos en profundidad y en amplitud, según la intención con la que dichos alumnos participen en la misma.

Llegados a este punto, es inevitable interrogarse sobre el origen de la intencionalidad con la que los alumnos abordan las actividades de aprendizaje - o, volviendo a la terminología que hemos introducido antes, sobre el origen del sentido que atribuyen a su participación en las mismas- y sobre los procesos psicológicos que intervienen en su formación. Aunque está, por supuesto, fuera del alcance de estas páginas intentar siquiera un resumen de lo que sabemos actualmente sobre factores y procesos implicados en la intencionalidad o sentido que los alumnos atribuyen a las actividades de aprendizaje, todo parece indicar que son numerosos y complejos y que en este punto, como en tantos otros, conviene huir de las explicaciones simples y excesivamente esquemáticas. Entwistle y sus colaboradores, por ejemplo, han puesto de relieve que existe una cierta relación entre el tipo de motivación y los enfoques de aprendizaje que adoptan los alumnos en una actividad determinada de aprendizaje. La motivación intrínseca, es decir, un elevado grado de interés por el contenido y por su relevancia, suele ir asociada con el enfoque en profundidad; cuando lo que predomina es el deseo de éxito, o la motivación por el logro, el enfoque de aprendizaje suele ser de tipo superficial; finalmente, si el motivo dominante es el miedo al fracaso, cabe esperar un enfoque de aprendizaje de tipo estratégico.

Ahora bien, la motivación de un alumno ante una actividad concreta de aprendizaje es a su vez el resultado de una serie de procesos que es necesario indagar. Apelar a la motivación sin más no ofrece una explicación satisfactoria. La manera como el profesor presenta la tarea y, sobre todo, la interpretación que de ello hace el alumno en función de factores tales como su autoconcepto académico, sus hábitos de trabajo y de estudio, sus estilos de aprendizaje, etc., son, sin du$\mathrm{da}$, algunos de los elementos claves a tener en cuenta. El hecho importante a destacar, sin embargo, es que esta interpretación tiene un carácter dinámico, no viene dada de una vez por todas, sino que se forja y se modifica en el transcurso mismo de la actividad de aprendizaje.

Quiere esto decir que el sentido que los alumnos atribuyen a una tarea escolar, y en consecuencia, los significados que pueden construir al respecto, no están determinados únicamente por sus conocimientos, habilidades, capacidades o experiencias previas, sino también por la compleja dinámica de intercambios comunicativos que se establecen a múltiples niveles entre los participantes, entre los propios alumnos y, muy especialmente, entre el profesor y los alumnos. Mediante el juego de las representaciones mutuas, de las expectativas que se generan, de los comportamientos a que éstas dan lugar, del intercambio de informaciones, del establecimiento más o menos explícito y consensuado de las reglas o normas de actuación, en suma, mediante el juego de los procesos psicosociológicos presentes en la situación de enseñanza, se va definiendo progresiva y conjuntamente el contexto en cuyo marco el alumno atribuye un sentido a lo que hace y construye unos significados, es decir, realiza unos aprendizajes con un determinado grado de significatividad.

\section{ENSEÑAR Y APRENDER, CONSTRUIR Y COMPARTIR}

La línea general de la argumentación que hemos desarrollado puede condu- 


\section{0}

cir fácilmente a inferencias erróneas - aunque ciertamente habituales y hasta cierto punto comprensibles cuando se hace un uso restrictivo y reduccionista del concepto de aprendizaje significativo- acerca de la manera de entender, planificar y llevar a cabo la enseñanza. En efecto, al poner de relieve la importancia de los procesos de pensamiento del alumno como elemento mediador entre la enseñanza y los resultados del aprendizaje, se hace necesario revisar la vieja creencia de que estos últimos son la consecuencia directa de la primera. La idea esencial de la tesis constructiva que subyace al concepto de aprendizaje significativo es, como ya hemos mencionado, que el aprendizaje que lleva a cabo el alumno no puede entenderse únicamente a partir de un análisis externo y objetivo de lo que le enseñamos y de cómo se lo enseñamos, sịno que es necesario tener en cuenta, además, las interpretaciones subjetivas que el propio alumno construye a este respecto. De aquí a poner en duda la posibilidad misma de enseñar en sentido estricto, a afirmar que la enseñanza debe renunciar a ejercer una influencia directa sobre el aprendizaje de los alumnos, a postular que debe limitarse a poner a éstos en contacto con los contenidos de aprendizaje para que puedan descubrir, inventar o construir los significados correspondientes, etc., sólo hay un paso, pero es un paso que a nuestro juicio no debe darse y que, si se da, equivale a vaciar el concepto de aprendizaje significativo de la mayor parte de su potencialidad heurística como instrumento de análisis y de reflexión psicopedagógica.

El error que se comete al dar el paso consiste en olvidar que los significados que los alumnos construyen en el transcurso de las actividades escolares no son significados cualesquiera, sino que corresponden a contenidos que en su mayor parte son de hecho creaciones culturales. En efecto, la práctica totalidad de los contenidos que intenta vehicular la educación escolar - desde los sistemas conceptuales y explicativos que configuran las disciplinas académicas tradicionales, hasta los métodos de trabajo, técnicas, habilidades y estrategias cognitivas, y por supuesto los valores, normas, actitudes, costumbres, modos de vida, etc.son formas culturales que tanto los profesores como los alumnos encuentran ya en buena parte elaborados y definidos antes de iniciar el proceso educativo. Aceptar este hecho en todas sus consecuencias implica, en palabras de Edward y Mercer (1987), «abandonar una perspectiva individualista sobre el desarrollo del conocimiento y de la comprensión y adoptar en su lugar un punto de vista psicológico que otorga la prioridad a la cultura y la comunicación».

En esta coyuntura, el problema que se plantea desde el punto de vista de la enseñanza y del aprendizaje significativo es doble. Por una parte, el alumno construye significados relativos a los contenidos escolares y la propia dinámica de este proceso constructivo dificulta o imposibilita los intentos de transmitírselos de una forma directa y acabada; pero, por otra parte, la naturaleza cultural de los contenidos marca la dirección en la que la enseñanza debe orientar, de forma progresiva, la construcción de significados. Los significados que finalmente construye el alumno son, pues, el resultado de una compleja serie de interacciones en las que intervienen como mínimo tres elementos: el propio alumno, los contenidos de aprendizaje y el profesor. Ciertamente, el alumno es el responsable último del aprendizaje en la medida en que construye su conocimiento atribuyendo sentido y significado a los contenidos de la enseñanza, pero es el profesor el que determina con su actuación, con su enseñanza, que las actividades en las que participa el alumno posibiliten un mayor o menor grado de amplitud y profundidad de los significados construidos y, sobre todo, 


\section{1}

el que asume la responsabilidad de orientar esta construcción en una determinada dirección.

Cuando se despoja el concepto de aprendizaje significativo de sus connotaciones más individualistas y se acepta que la atribución de sentidos y la construcción de significados en el ámbito escolar - y muy probablemente en cualquier otro ámbito de la vida humana-, son procesos fuertemente impregnados y orientados por las formas culturales y que, por lo tanto, tienen lugar necesariamente en un contexto de relación y de comunicación interpersonal que trasciende ampliamente la dinámica interna de los procesos de pensamiento de los alumnos; cuando se acepta este planteamiento, la tesis constructivista aplicada al aprendizaje escolar adquiere una nueva dimensión. En efecto, la construcción del conocimiento es, en esta perspectiva, una construcción claramente orientada a compartir significados y sentidos, mientras que la enseñanza es un conjunto de actividades sistemáticas y planificadas mediante las cuales profesor y alumno llegan a compartir parcelas progresivamente más amplias de significados respecto a los contenidos del curriculum escolar.

Es evidente que en esta construcción progresiva de significados compartidos, el profesor y el alumno juegan papeles netamente distintos: el profesor conoce en principio los significados que espera llegar a compartir con el alumno al término del proceso educativo y este conocimiento le sirve para planificar la enseñanza; el alumno, por el contrario, desconoce este referente último - si lo conociera no tendría sentido su participación en el acto de enseñanza formalhacia el que trata de conducirle el profesor y, por lo tanto, debe ir acomodando progresivamente los sentidos y significados que construye de forma ininterrumpida en el transcurso de las actividades o tareas escolares. En otros términos, el profesor guía el proceso de construcción de conocimiento del alumno haciéndole participar en tareas y actividades que le permitan construir significados cada vez más próximos a los que poseen los contenidos del curriculum escolar. El profesor es, pues, al mismo tiempo un guía y un mediador. Estamos muy lejos, en consecuencia, de las concepciones de la enseñanza que mencionábamos al comienzo de este apartado.

Esta visión del proceso de enseñanza/aprendizaje supone un vuelco respecto a algunas utilizaciones hàbituales del concepto de aprendizaje significativo y plantea nuevas y apasionantes cuestiones sobre los mecanismos a través de los cuales se ejerce la influencia educativa; es decir, sobre los mecanismos que hacen posible que el profesor enseñe, que el alumno aprenda y construya su propio conocimiento, y que ambos lleguen a compartir, en mayor o menor grado, que congrega actualmente los esfuerzos de un buen número de investigadores en psicología y educación, excede ya los objetivos que nos habíamos fijado.

Nuestro propósito era simplemente mostrar que el concepto de aprendizaje significativo es un instrumento útil y valioso para el análisis y la reflexión psicopedagógica. Sin embargo, los argumentos y comentarios que hemos ido articulando en las páginas anteriores indican que, para que pueda desempeñar adecuadamente este papel, es necesario despojarlo de muchas de las connotaciones que ha ido acumulando de forma subrepticia $y$, al mismo tiempo, desarrollar otras que hasta el momento han sido escasamente tomadas en consideración. Particularmente importante nos parece, en concreto, atender tanto al sentido como ai significado del aprendizaje escolar; renunciar a las connotaciones más individualistas del proceso de construcción de significados y sentidos; y, por úl- 
timo, resituar este proceso de construcción en el contexto de relación y comunicación interpersonal que es intrínseco al acto de enseñanza.

\section{Referencias}

Ausubel. D. P; Novak. J. D.; Hanesian, H. (1983). Psicología Educativa. México: Trillas [Educational Psychology. Nueva York: Holt, Rinehart \& Wiston].

BRUNER, J. S. (1961). The act of discovery. Harvard Educational Review, 31, 21-32.

EDWARDS, D.; MERCER, N. (1987). Common Knowledge. The development of understanding in the classroom. Londres: Methuen.

ENTWISTIE, N. (1987). Understanding classroom learning. Londres: Hodder and Stoughton.

ENTWISTIE, N. J.; RAMSDEN, P. (1983). Understanding student learning. Londres: Croom Helm.

FARNHAM-DIGGORY, S. (1972). Cognitive processes in education: a psychological preparation for teaching and curriculum. Nueva York: Harper and Row.

MARTON, F. (1981). Phenomenography: describing conceptions of the world around us. Instructional Science, 10, 177-220.

MARTON, F. (1983). Beyond individual differences. Educational Psychology, 3, 289-304.

MaSLOW, A. (1968). Some educational implications oh humanistic psuchologies. Harvard Educational Review, 38, 685-696.

Nơ, L. (1979). Les pédagogies de la connaissance. Tolouse: Privat.

Novak. J. D. (1982). Teoría y práctica de la educación. Madrid: Alianza [A theory of Education. Cornell: Cornell University Press, 1977].

PIAGET, J. (1974). A dónde va la educación. Barcelona: Teide [Où va l'Education? Paris: Denöel/Gonthier, 1972].

PLA. L. (1987). Bases psicopedagògiques per a l'ensenyament de l'anglès. Tesis doctoral no publicada. Universidad de Barcelona.

RoGers, C. R. (1975). Libertad y creatividad en la educación. Buenos Aires: Paidós [Freedom to learn. Columbus, Ohio: Ch. E. Merril, 1969].

WITTROCK, M. C. (1974). Learning as a generative process. Educational Psychologist, 11, 87-95.

WITTROCK, M. C. (1986). Student's thought Processes. En M. C. Wittrock (Ed.). Handbook of research on teaching. Nueva York: MacMillan, PP. 297-314. 